\title{
Homological thickness and stability of torus knots
}

\author{
MARKO STOŠIĆ
}

\begin{abstract}
In this paper we show that the nonalternating torus knots are homologically thick, ie that their Khovanov homology occupies at least three diagonals. Furthermore, we show that we can reduce the number of full twists of the torus knot without changing certain part of its homology, and consequently, there exists stable homology of torus knots conjectured by Dunfield, Gukov and Rasmussen in [3]. Since our main tool is the long exact sequence in homology, we have applied our approach in the case of the Khovanov-Rozansky $s l(n)$ homology, and thus obtained analogous stability properties of $s l(n)$ homology of torus knots, also conjectured in [3].
\end{abstract}

$57 \mathrm{M} 25$

\section{Introduction}

In recent years there has been a lot of interest in the "categorification" of link invariants, initiated by M Khovanov in [6]. For each link $L$ in $S^{3}$ he defined a graded chain complex with grading-preserving differentials whose graded Euler characteristic is equal to the Jones polynomial of the link $L$ (see Jones [4] and Kauffman [5]) and whose homology groups (usually called $s l(2)$ homology groups) are link invariants. This is done by starting from the state-sum expression for the Jones polynomial (which is written as an alternating sum), then constructing for each term a graded module whose graded dimension is equal to the value of that term, and finally, defining the differentials as appropriate grading preserving maps, so that the complex obtained is a link invariant (up to chain homotopy).

Although the theory is rather new, it already has strong applications in low-dimensional topology, for instance, the short proof of the Milnor conjecture by Rasmussen in [12], as well as his proof of the existence of exotic differential structures on $\mathbb{R}^{4}$ [14], which were previously accessible only by gauge theory.

The advantage of Khovanov homology theory is that its definition is combinatorial and since there is a straightforward algorithm for computing it, it is (theoretically) highly calculable. Nowadays there are several computer programs such as The Knot Atlas [1] and KhoHo [16] that can calculate effectively Khovanov homology of links with up to 50 crossings. 
Based on the calculations there are many conjectures about the properties of link homology; see eg Bar-Natan [2], Khovanov [7] and Dunfield, Gukov and Rasmussen [3]. Some of the properties have been verified (see Lee [10; 9]), but many of them are still open.

In this paper we first show that the torus knots $T_{p, q}$ for $3 \leq p \leq q$ (nonalternating torus knots) are homologically thick, ie that their Khovanov homology occupies at least three diagonals. Furthermore, we obtain even stronger results that relate the homology of the torus knots $T_{p, q}$ and $T_{p, q+1}$. Namely, we prove that, up to a certain homological degree, their (unnormalized) homologies coincide.

As the first application of this result we calculate the homology of torus knots for low homological degrees. We also obtain the proof of the existence of stable Khovanov homology of torus knots, conjectured by Dunfield, Gukov and Rasmussen in [3].

Furthermore, we conjecture that the homological width of the torus knot $T_{p, q}$ is at least $p$, and we reduce this problem to determining the nontriviality of certain homological groups.

An analogous categorification of the $n$-specializations of the HOMFLYPT polynomial was carried out by M Khovanov and L Rozansky in 2004 [8]. The construction uses the state-sum model for the HOMFLYPT polynomial [11] and is analogous to the categorification of the Jones polynomial: it uses the same cubic complex construction, and there exists a similar long exact sequence in homology. However, since the state-sum model for the HOMFLYPT polynomial is much more complicated than Kauffman's state-sum model, the explicit calculation of the homology groups is very hard. Consequently, the values of the $s l(n)$ link homology are known only for a very small class of knots - two-bridge knots by Rasmussen [13] and the closures of certain three-strand braids by Webster [21].

Since in the proofs of results for Khovanov homology of torus knots, we mainly use the long exact sequence of Khovanov homology (3), we also obtain most of the analogous results for the stability of $s l(n)$ homology of torus knots by using the long exact sequence in the $s l(n)$ homology (13). Namely, we prove that up to a certain homological degree, the (unnormalized) $s l(n)$ homology groups of $T_{p, q}$ and $T_{p, q-1}$ torus knots coincide and, consequently, that there exists stable $s l(n)$ homology of torus knots, also conjectured in [3].

The organization of the paper is as follows: in Section 2 we recall briefly the definition and the basic properties of Khovanov homology and introduce notation for positive braid knots and torus knots. In Section 3 we give the statements of the results that we obtain concerning Khovanov homology of torus knots. More precisely, in Section 3.1, 
we relate the homologies of the torus knots $T_{p, q}$ and $T_{p, q+1}$, we show that the torus knots are homologically thick and we calculate the homology groups of torus knots in the homological degrees $0,1,2,3$ and 4. In Section 3.2 we conjecture further results concerning the thickness of torus knots, and in Section 3.3 we show that there exists stable Khovanov homology for torus knots. Section 4 contains the proofs of theorems from Section 3. Finally, in Section 5, we a give short introduction to KhovanovRozansky $(s l(n))$ homology, and we prove that there exists stable $s l(n)$ homology of torus knots.

Acknowledgements The author would like to thank M Khovanov and J Rasmussen for many helpful discussions and suggestions. This work was supported by the Programa Operacional 'Ciência e Inovação 2010, project number POCI/MAT/60352/2004, financed by the Fundação para a Ciência e a Tecnologia (FCT) and cofinanced by the European Community fund FEDER. While this work was carried out the author was at the Departamento de Matemática, IST, Lisbon, and was supported by Fundação de Ciência e Tecnologia/(FCT), grant no SFRH/BD/6783/2001.

\section{Notation}

\subsection{Khovanov ( $\operatorname{sl}(2))$ homology}

We recall briefly the definition of Khovanov homology for links. For more details see Bar-Natan [2] and Khovanov [6]. First of all, take a link $K$, its planar projection $D$, and take an ordering of the crossings of $D$. For each crossing $c$ of $D$, we define 0-resolution $D_{0}$ and 1-resolution $D_{1}$, as in the figure below.
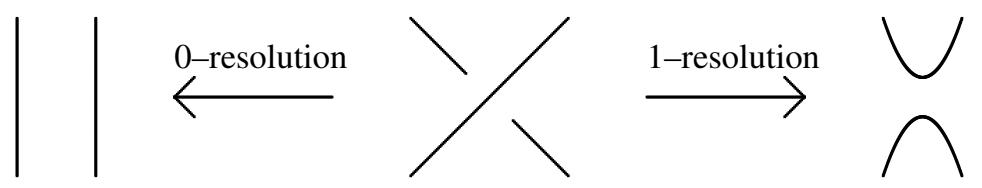

Denote by $m$ the number of crossings of $D$. Then there is bijective correspondence between the total resolutions of $D$ and the set $\{0,1\}^{m}$. Namely, to every $m$-tuple $\epsilon=\left(\epsilon_{1}, \ldots, \epsilon_{m}\right) \in\{0,1\}^{m}$ we associate the resolution $D_{\epsilon}$ where we resolved the $i-$ th crossing in a $\epsilon_{i}$-resolution. Every resolution $D_{\epsilon}$ is a collection of disjoint circles. To each circle we associate graded $\mathbb{Z}$-module $V$, which is freely generated by two basis vectors 1 and $X$, with $\operatorname{deg} \mathbf{1}=1$ and $\operatorname{deg} X=-1$. To $D_{\epsilon}$ we associate the module $M_{\epsilon}$, which is the tensor product of $V$ 's over all circles in the resolution. Now, all the 
resolution $D_{\epsilon}$ with fixed $|\epsilon|$ (sum of elements of $\epsilon$ ) are grouped, and all resolutions are drawn as (skewed) $m$-dimensional cube such that in $i$-th column are the resolutions $D_{\epsilon}$ with $|\epsilon|=i$, as in the following picture:

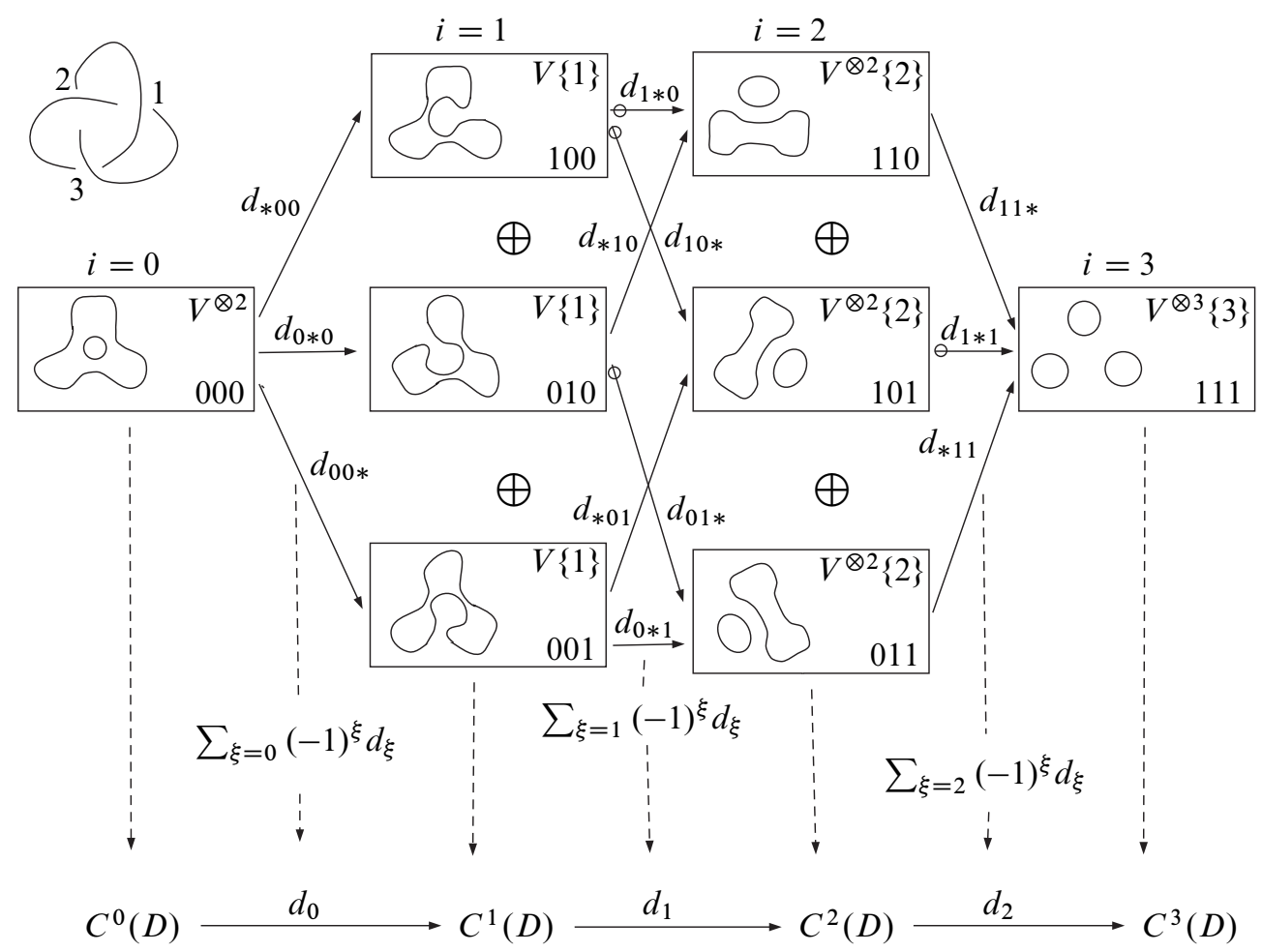

Figure 1: The chain complex and the differentials in the case of the trefoil knot

The $i$-th chain group $C^{i}$ is given by

$$
C^{i}(D)=\oplus_{|\epsilon|=i} M_{\epsilon}\{i\} .
$$

Here, by $\{i\}$, we have denoted the upward shift by $i$ in the grading of $M_{\epsilon}$ (for more details see eg Bar-Natan [2]). The differential $d^{i}: C^{i}(G) \rightarrow C^{i+1}(G)$ is defined as (signed) sum of "per-edge" differentials. Namely, the only nonzero maps are from $D_{\epsilon}$ to $D_{\epsilon^{\prime}}$, where $\epsilon=\left(\epsilon_{1}, \ldots, \epsilon_{m}\right), \epsilon_{i} \in\{0,1\}$, if and only if $\epsilon^{\prime}$ has all entries same as $\epsilon$ except one $\epsilon_{j}$, for some $j \in\{0,1\}$, which is changed from 0 to 1 . We denote these differentials by $d_{\nu}$, where $v$ is $m$-tuple which consists of the label $*$ at the position $j$ and of $m-10$ 's and 1's (the same as the remaining entries of $\epsilon$ ). Note that in these cases, either two circles of $D_{\epsilon}$ merge into one circle of $D_{\epsilon^{\prime}}$ or one circle of $D_{\epsilon}$ splits into two circles of $D_{\epsilon^{\prime}}$, and all other circles remain the same. In the first case, the map $d_{v}$ is defined as the identity on the tensor factors $(V)$ that correspond to the unchanged 
circles, and on the remaining factors is given as the (graded preserving) multiplication map $m: V \otimes V \rightarrow V\{1\}$, which is given on basis vectors by

$$
m(\mathbf{1} \otimes \mathbf{1})=1, \quad m(\mathbf{1} \otimes X)=m(X \otimes \mathbf{1})=X, \quad m(X \otimes X)=0 .
$$

In the second case, the map $d_{v}$ is defined as the identity on the tensor factors $(V)$ that correspond to the unchanged circles, and on the remaining factors is given as the (graded preserving) comultiplication map $\Delta: V \rightarrow V \otimes V\{1\}$, which is given on basis vectors by

$$
\Delta(\mathbf{1})=\mathbf{1} \otimes X+X \otimes \mathbf{1}, \quad \Delta(X)=X \otimes X .
$$

Finally, to obtain the differential $d^{i}$ of the chain complex $C(D)$, we sum all contributions $d_{v}$ with $|v|=i$, multiplied by the sign $(-1)^{f(v)}$, where $f(v)$ is equal to the number of 1 's ordered before $*$ in $v$. This makes every square of our cubic complex anticommutative, and hence we obtain the genuine differential (ie $\left(d^{i}\right)^{2}=0$ ).

The homology groups of the obtained complex $(C(D), d)$ we denote by $H^{i}(D)$ and call unnormalized homology groups of $D$. In order to obtain link invariants (ie independence of the chosen projection), we have to shift the chain complex (and hence the homology groups) by

$$
\mathcal{C}(D)=C(D)\left[-n_{-}\right]\left\{n_{+}-2 n_{-}\right\},
$$

where $n_{+}$and $n_{-}$are the numbers of positive and negative crossings, respectively, of the diagram $D$ (see below for conventions).

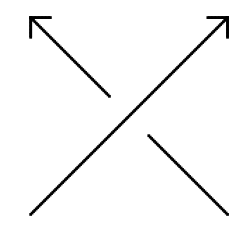

positive

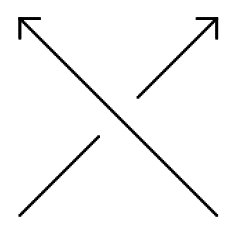

negative

In the formula (1), we have denoted by $\left[-n_{-}\right]$, the shift in homology degrees [2]. The homology groups of the complex $\mathcal{C}(D)$ we denote by $\mathcal{H}^{i}(D)$. Hence, we have

$$
\mathcal{H}^{i, j}(D)=H^{i+n_{-}, j-n_{+}+2 n_{-}}(D)
$$

Theorem 2.1 [6;2] The homology groups $\mathcal{H}(D)$ are independent of the choice of the planar projection $D$. Furthermore, the graded Euler characteristic of the complex $\mathcal{C}(D)$ is equal to Jones polynomial of the link $K$.

Hence, we can write $\mathcal{H}(K)$, and we call $\mathcal{H}^{i}(K)$ the homology groups of the link $K$. 
Let $D$ be a diagram of a link $K$ and let $c$ be one of its crossings. Denote by $D_{i}$, $i=0,1$ the diagram that is obtained after performing an $i$-resolution of the crossing $c$. Then one can see that the complex $C(D)$ is in fact the mapping cone of a certain homomorphism $f: C\left(D_{0}\right) \rightarrow C\left(D_{1}\right)$ (which is basically given by maps $m$ and $\Delta$ ). Hence, there exists a long exact sequence of (unnormalized) homology groups (see eg Viro [20]):

$$
\begin{aligned}
& \cdots \rightarrow H^{i-1, j-1}\left(D_{1}\right) \rightarrow H^{i, j}(D) \rightarrow \\
& \qquad H^{i, j}\left(D_{0}\right) \rightarrow H^{i, j-1}\left(D_{1}\right) \rightarrow H^{i+1, j}(D) \rightarrow \cdots
\end{aligned}
$$

This long exact sequence is the categorification of the defining recursive relation of the Kauffman bracket:

$$
\langle D\rangle=\left\langle D_{0}\right\rangle-q\left\langle D_{1}\right\rangle
$$

Indeed, this relation can be obtained by taking the graded Euler characteristic of (3).

A link $K$ is called a positive link, if it has a planar projection with only positive crossings. Then we have the following:

Lemma 2.2 If $K$ is a positive link, then $\mathcal{H}^{i}(K)$ is trivial for all $i<0$. Furthermore, if $D$ is the planar projection of positive knot $K$, with $n_{-}$negative crossings, then $H^{i}(D)$ is trivial for $i<n_{-}$.

Proof Let $\bar{D}$ be a planar projection of link $K$, with all crossings positive. Then from the definition of the cubic complex, we have that $C^{i}(\bar{D})$ are trivial for $i<0$, and so $H^{i}(\bar{D})$ are trivial for $i<0$. Thus, from (2), we have that $\mathcal{H}^{i}(K)$ is trivial for all $i<0$.

If $D$ is the planar projection of the link $K$, with $n_{-}$negative crossings, then from (2) we have that $H^{i}(D)$ is, up to degree shift, equal to $\mathcal{H}^{i-n_{-}}(K)$, and hence is trivial when $i-n_{-}<0$, as wanted.

Usually, the homology groups of the link $K$ are represented as a planar array in such a way that $\operatorname{rank} \mathcal{H}^{i, j}(K)=\operatorname{dim}\left(\mathcal{H}^{i, j}(K) \otimes \mathbb{Q}\right.$ ) (or the whole group $\mathcal{H}^{i, j}(K)$, if we want to keep track of the torsions) is specified in the position $(i, j)$. As can be seen from the definitions, the $q$-gradings $(j)$ of the generators of nontrivial $\mathcal{H}^{i, j}(K)$ and the number of components of $K$ are of the same parity (either all are even or all are odd) [6, Proposition 30]. Hence, by a diagonal of the homology of the link $K$, we mean a line $j-2 i=a=\mathrm{const}$, when there exist integers $i$ and $j$ such that $j-2 i=a$ and $\operatorname{rank} \mathcal{H}^{i, j}(K)>0$. If $a_{\max }$ and $a_{\min }$ are the maximal and minimal value of $a$ such that the line $j-2 i=a$ is a diagonal of the homology of the link $K$, then we define the homological width of the link $K$ to be $h(K)=\left(a_{\max }-a_{\min }\right) / 2+1$. Every knot (link) 
occupies at least two diagonals, ie $h(K) \geq 2$ for every link $K$, since the homology group $\mathcal{H}^{0}(K)$ has at least two generators in the $q$-degrees $s(K)-1$ and $s(K)+1$, for certain integer $s(K)[10 ; 12]$. Knots that occupy exactly two diagonals are called $\mathrm{H}$-thin, or homologically thin. For example all alternating knots are H-thin [10], and the free part of the homology of any H-thin knot is determined by its Jones polynomial and the signature. A knot that is not $\mathrm{H}$-thin is called $\mathrm{H}$-thick or homologically thick. Furthermore, for an element $x \in \mathcal{H}^{i, j}(K)$, we denote its homological grading $i$ by $t(x)$, and its $q$-grading (also called the quantum grading) $j$ by $q(x)$. We also introduce a third grading $\delta(x)$ by $\delta(x)=q(x)-2 t(x)$. Hence, we have that the knot $K$ is H-thick, if there exist three generators of $\mathcal{H}(K)$ with different values of the $\delta$-grading.

An alternative way of presenting the homology of the knot is by means of the twovariable Poincaré polynomial $P(K)(t, q)$ of the chain complex $\mathcal{C}(D)$ :

$$
P(K)(t, q)=\sum_{i, j \in \mathbb{Z}} t^{i} q^{j} \operatorname{rank} \mathcal{H}^{i, j}(K)
$$

\subsection{Positive braid knots}

The positive braid knots are the knots (or links) that are the closures of positive braids. Let $K$ be arbitrary positive braid knot and let $D$ be its planar projection which is the closure of a positive braid. Denote the number of strands of that braid by $p$. We say that the crossing $c$ of $D$ is of the type $\sigma_{i}, i<p$, if it corresponds to the generator $\sigma_{i}$ in the braid word of which $D$ is the closure. Denote the number of crossings of the type $\sigma_{i}$ by $l_{i}, i=1, \ldots, p-1$ and order them from top to bottom. Then each crossing $c$ of $D$ we can write as the pair $(i, \alpha)$ (we will also write $(i \alpha)$ if there is no possibility of confusion), $i=1, \ldots, p-1$ and $\alpha=1, \ldots, l_{i}$, if $c$ is of the type $\sigma_{i}$ and it is ordered as $\alpha$-th among the crossings of the type $\sigma_{i}$. Finally, we order the crossings of $D$ by the following ordering: $c=(i \alpha)<d=(j \beta)$ if and only if $i<j$, or $i=j$ and $\alpha<\beta$.

For some results on the homology of positive braid knots, see eg Stošić [18].

\subsection{Torus knots}

A knot or a link is a torus knot if it is isotopic to a knot or a link that can be drawn without any points of intersection on the trivial torus. Every torus link is, up to a mirror image, determined by two nonnegative integers $p$ and $q$, ie it is isotopic to a unique torus knot $T_{p, q}$ which has the diagram $D_{p, q}$ - the closure of the braid $\left(\sigma_{1} \sigma_{2} \ldots \sigma_{p-1}\right)^{q}$

- as a planar projection. In other words, $D_{p, q}$ is the closure of the $p$-strand braid with 
$q$ full twists. Note that the number of crossings of $D_{p, q}$ is $(p-1) q$, and that they are all positive. Since $T_{p, q}$ is isotopic to $T_{q, p}$ we can assume that $p \leq q$.

If $p=1$ then the torus knot $T_{p, q}$ is trivial and for $p=2$ the torus knots $T_{2, q}$ are alternating and their homology is well-known [6]. In particular, their homology occupies exactly two diagonals.

\section{Thickness and stability of Khovanov homology for torus knots}

In this section we give the overview of the results that we have obtained concerning the Khovanov homology of torus knots. All proofs are given in Section 4.

\subsection{Thickness of torus knots}

As we saw, if $p \leq 2$, torus knots $T_{p, q}$ are alternating, and their homology occupies exactly two diagonals. However, if $p \geq 3$, the torus knot $T_{p, q}$ is nonalternating, and we will prove that its homology occupies at least three diagonals.

In order to prove this, first we give more general theorem that allow us to reduce the number of full twists of the torus knot without affecting certain (unnormalized) homological groups. Namely, in Section 4 we prove the following theorem:

Theorem 3.1 Let $p, q$ and $i$ be integers such that $2 \leq p<q$ and $i<p+q-3$. Then for every $j \in \mathbb{Z}$,

$$
H^{i, j}\left(D_{p, q}\right)=H^{i, j}\left(D_{p, q-1}\right) .
$$

Furthermore, for every $2 \leq p<q$ and $i<2 p-1$ and $j \in \mathbb{Z}$, we have

$$
H^{i, j}\left(D_{p, p+1}\right)=H^{i, j}\left(D_{p, p+2}\right)=\cdots=H^{i, j}\left(D_{p, q}\right) .
$$

Also, for every $p \geq 2, i<2 p-3$ and $j \in \mathbb{Z}$, we have

$$
H^{i, j}\left(D_{p, p}\right)=H^{i, j+1}\left(D_{p-1, p}\right) .
$$

Remark 3.2 In fact, in the course of the proof, we have obtained the stronger version of (4). Namely, if $p$ and $q$ are integers such that $2 \leq p<q$ then for every $j \in \mathbb{Z}$, we have

$$
H^{i, j}\left(D_{p, q}\right)=H^{i, j}\left(D_{p, q-1}\right), \quad \text { for } \quad i<q-1+[(q-1) / p](p-2) .
$$


One of the first corollaries is the fact that the nonalternating torus knots are homologically thick.

Theorem 3.3 Every torus knot $T_{p, q}, p, q \geq 3$ is H-thick, ie its Khovanov homology occupies at least three diagonals.

The second corollary is the computation of the homology groups of $T_{p, q}$, for $3 \leq p<q$, with low homological degree.

Theorem 3.4 Let $3 \leq p<q$. Then we have

$$
\begin{aligned}
\mathcal{H}^{0,(p-1)(q-1) \pm 1}\left(T_{p, q}\right) & =\mathbb{Z} \\
\mathcal{H}^{2,(p-1)(q-1)+3}\left(T_{p, q}\right) & =\mathbb{Z} \\
\mathcal{H}^{3,(p-1)(q-1)+7}\left(T_{p, q}\right) & =\mathbb{Z} \\
\mathcal{H}^{3,(p-1)(q-1)+5}\left(T_{p, q}\right) & =\mathbb{Z}_{2} \\
\mathcal{H}^{4,(p-1)(q-1)+6 \pm 1}\left(T_{p, q}\right) & =\mathbb{Z} .
\end{aligned}
$$

All other $\mathcal{H}^{i, j}\left(T_{p, q}\right)$ for $i=0, \ldots, 4$, are trivial.

\subsection{Further thickness results}

As we shall see, Theorem 3.3 will follow from the following:

Proposition 3.5 Let $K=T_{p, q}, 3 \leq p \leq q$ be a torus knot. Then

$$
\operatorname{rank} \mathcal{H}^{4,(p-1)(q-1)+5}(K)>0 .
$$

Even though we have shown that the torus knots $T_{p, q}, p \geq 3$, are H-thick, from the existing experimental results one can see that the homology of torus $T_{p, q}$ knots occupies at least $p$ diagonals (ie that its homological width is at least $p$ ). In fact, one can see that in all examples we have that $H^{2 p-2, p}\left(D_{p, q}\right)$ is of nonzero rank.

Proposition 3.6 If rank $H^{2 p-2, p}\left(D_{p, q}\right)>0$ then the homological width of the torus knot $T_{p, q}$ is at least $p$.

Thus, to show that the Khovanov homology of torus knot $T_{p, q}$ occupies at least $p$ diagonals, we are left with proving that rank $H^{2 p-2, p}\left(D_{p, q}\right)>0$. From (5) we have that $H^{2 p-2, p}\left(D_{p, q}\right)=H^{2 p-2, p}\left(D_{p, p+1}\right)$. Furthermore we have:

Lemma 3.7 $H^{2 p-2, p}\left(D_{p, p}\right)=H^{2 p-2, p}\left(D_{p, p+1}\right)$. 
Conjecture 3.8 The rank of the homology group $H^{2 p-2, p}\left(D_{p, p}\right)$ (and equivalently of $\left.H^{2 p-2, p}\left(D_{p, p+1}\right)\right)$ is nonzero.

As we saw, the validity of Conjecture 3.8 implies that the homological width of the torus knot $T_{p, q}$ is at least $p$. Even though we don't (yet) have the proof of Conjecture 3.8, there is evidence that it is true. First of all, the computer program calculations show that the conjecture is true at least for $p \leq 7$ (the calculations are mainly for knots, ie for $\left.D_{p, p+1}\right)$. Furthermore, Lee's variant $H_{L}^{i, j}$ of Khovanov homology [9] for the $p$-component link $D_{p, p}$ has $2 p$ generators in the homological degree $2 p-2$. Also, as it is well-known, there exist spectral sequences whose $E_{\infty}$-page is Lee's homology and whose $E_{2}-$ page is Khovanov homology (see Rasmussen [12] and Turner [19]). So $H_{L}^{i, j} \subset H^{i, j}$ and hence $H^{2 p-2}\left(D_{p, p}\right)$ has at least $2 p$ generators. So, we are left with proving that at least one of them has the $q$-grading equal to $p$.

\subsection{Stability of homology of torus knots}

In Theorem 3.1, we have proved that we can reduce the number of full twists, $q$, of the standard diagram $D_{p, q}$ of the torus knot $T_{p, q}$ without changing the first $p+q-3$ homology groups. In other words, we have obtained the existence of stable homology of torus knots, conjectured in [3].

We can write this down formally in the following way. Define the following normalization of the Poincaré polynomial of the homology of the torus knot:

$$
P_{m, n}(t, q)=q^{-(m-1) n} P\left(T_{m, n}\right)(t, q) .
$$

Then from the "descending" properties of Theorem 3.1 we have the following:

Theorem 3.9 For every $m \in \mathbb{N}$ there is a stable homology polynomial $P_{m}^{S}$ given by

$$
P_{m}^{S}(t, q)=\lim _{n \rightarrow \infty} P_{m, n}(t, q) .
$$

Furthermore, as we have shown, the (normalized) Poincaré polynomial $P_{m, n}(t, q)$ of the torus knot $T_{m, n}$ coincides with the stable polynomial $P_{m}^{S}$, for all powers of $t$ up to $m+n-3$ (even up to $n-1+[(n-1) / m](m-2)$, according to Remark 3.2). Similar results are obtained at the conjectural level in [3] (with a conjectural bound on the powers of $q$ for agreement between the stable homology and the effective homology of any particular torus knot). In [3], reduced homology [7] is used, but the whole method and all proofs work in the same way for reduced homology. As we shall see in Section 5, the analogous stability properties are valid in the case of Khovanov-Rozansky homology. 


\section{Proofs}

\subsection{Proof of Theorem 3.1}

First we shall prove the "descending" property (4). In order to do this we will use the long exact sequence (3) and we will relate the unnormalized $i$-th homology groups of the standard regular diagrams of the torus knots $D_{p, q}$ and $D_{p, q-1}$ for $p<q$.

Let $3 \leq p<q$. Let $c_{p-1}$ be the crossing $(p-1,1)$ of the diagram $D_{p, q}$. Now denote by $E_{p, q}^{1}$ and $D_{p, q}^{1}$ the 1- and 0-resolutions, respectively, of the diagram $D_{p, q}$ at the crossing $c_{p-1}$. Then from (3) we obtain the following long exact sequence:

$$
\begin{aligned}
& \cdots \rightarrow H^{i-1, j-1}\left(E_{p, q}^{1}\right) \rightarrow H^{i, j}\left(D_{p, q}\right) \rightarrow \\
& H^{i, j}\left(D_{p, q}^{1}\right) \rightarrow H^{i, j-1}\left(E_{p, q}^{1}\right) \rightarrow H^{i, j}\left(D_{p, q}\right) \rightarrow \cdots
\end{aligned}
$$

Now, we can continue the process, and resolve the crossing $c_{p-2}=(p-2,1)$ of $D_{p, q}^{1}$ in two possible ways. Denote the diagram obtained by the 1 -resolution by $E_{p, q}^{2}$, and the diagram obtained by the $0-$ resolution by $D_{p, q}^{2}$. Then from (3) we have the long exact sequence:

$$
\begin{aligned}
\cdots \rightarrow H^{i-1, j-1}\left(E_{p, q}^{2}\right) \rightarrow H^{i, j}\left(D_{p, q}^{1}\right) \rightarrow & \\
& H^{i, j}\left(D_{p, q}^{2}\right) \rightarrow H^{i, j-1}\left(E_{p, q}^{2}\right) \rightarrow H^{i+1, j}\left(D_{p, q}^{1}\right) \rightarrow \cdots
\end{aligned}
$$

After repeating this process $p-1$ times (resolving the crossing $c_{p-k}=(p-k, 1)$ for $k=1, \ldots, p-1$ of $D_{p, q}^{k-1}$, obtaining the 1 -resolution $E_{p, q}^{k}$ and 0 -resolution $D_{p, q}^{k}$ and applying the same long exact sequence in homology), we obtain that for every $k=1, \ldots, p-1$, the following sequence is exact:

$$
\begin{aligned}
\cdots \rightarrow H^{i-1, j-1}\left(E_{p, q}^{k}\right) \rightarrow & H^{i, j}\left(D_{p, q}^{k-1}\right) \rightarrow \\
& H^{i, j}\left(D_{p, q}^{k}\right) \rightarrow H^{i, j-1}\left(E_{p, q}^{k}\right) \rightarrow H^{i+1, j}\left(D_{p, q}^{k-1}\right) \rightarrow \cdots
\end{aligned}
$$

Here $D_{p, q}^{0}$ denotes $D_{p, q}$, and we obviously have that $D_{p, q}^{p-1}=D_{p, q-1}$. Our goal is to show that $H^{i}\left(E_{p, q}^{k}\right)$ are trivial for every $i<p+q-3$ and $0<k<p$. This is done by using the following lemma.

Lemma 4.1 For every three positive integers $p, q$ and $k$, such that $3 \leq p<q$ and $k<p$, the knot with the diagram $E_{p, q}^{k}$ is positive, and the diagram $E_{p, q}^{k}$ has at least $p+q-3$ negative crossings.

Proof Since for every $0<k<p, E_{p, q}^{k}$ is obtained by the 1-resolution of the crossing $c_{p-k}=(p-k, 1)$ of the (positive braid knot) diagram $D_{p, q}^{k-1}$, it is the closure of the plat braid diagram with only one plat $E_{p-k}$ : 


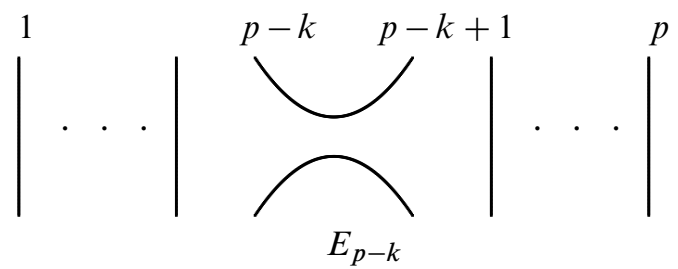

Now, note that two lower strands of $E_{p-k}$ are always "neighbor" strands, ie they form a ribbon, through the diagram, until they reach the upper part of $E_{p-k}$. So, we can "slide" the lower part of the plat $E_{p-k}$ through the diagram (by using the second Reidemeister move R2 and also the first Reidemeister move R1 where the two strands intersect each other) until it reaches the left or the right hand side of the upper part of $E_{p-k}$. If it first reaches the right hand side, it automatically (or after a R1 move) becomes the closure of a positive braid diagram. If it first reaches the left hand side then after a R1 move and a "slide" (sequence of R2 moves) we obviously obtain a positive braid diagram.

Concerning the number of negative crossings of $E_{p, q}^{k}$, note that by performing the first sequence of R2 moves (sliding the lower part of $E_{p-k}$ through the diagram, from the top to the bottom) in each move we have "canceled" one positive and one negative crossing. Furthermore, since $p<q$, the two strands of the lower part of the plat $E_{p-k}$ will make a full twist at least once, and so they will have two crossings with each other, which are both obviously negative crossings. So, we have that on each of the last (lower) $q-1$ blocks $\left(\sigma_{1} \ldots \sigma_{p-1}\right)$ of $E_{p, q}^{k}$ we have at least one negative crossing. Furthermore, on the part where both lower strands of the plat $E_{p-k}$ make full twists, we have applied $p-2 \mathrm{R} 2$ moves, and hence we have in addition, at least, $p-2$ negative crossings. Altogether, this gives at least $q-1+p-2=p+q-3$ negative crossings of $E_{p, q}^{k}$, as required.

Remark 4.2 Obviously, the two "neighboring" strands from Lemma 4.1 will make at least $[(q-1) / p]$ full twists, where by $[x]$ we have denoted the largest integer not greater than $x$. So, in fact we have proved that the diagram $E_{p, q}^{i}$ has at least $q-1+[(q-1) / p](p-2)$ negative crossings.

Now, we can go back to the proof of Theorem 3.1. From Lemma 4.1 and Lemma 2.2, we conclude that for every $0<k<p$,

$$
H^{i, j}\left(E_{p, q}^{k}\right) \text { is trivial for } i<p+q-3 .
$$

Thus, from (8) and (9), we obtain that for $i<p+q-3$ and every $j \in \mathbb{Z}$,

$$
H^{i, j}\left(D_{p, q}^{k-1}\right)=H^{i, j}\left(D_{p, q}^{k}\right), \quad k=1, \ldots, p-1,
$$

Algebraic 83 Geometric Topology, Volume 7 (2007) 
and thus we have that

$$
H^{i, j}\left(D_{p, q}\right)=H^{i, j}\left(D_{p, q-1}\right), \quad \text { for } i<p+q-3,
$$

which gives (4). Moreover, by Remark 4.2, in fact we have obtained (7).

Formula (5) obviously follows from (4) since $i<2 p-1=p+(p+2)-3$.

To obtain (6) apply the set of long exact sequences (8) for the case $p=q$. In this case, like in Lemma 4.1, we obtain that $E_{p, p}^{k}$ is the diagram of a positive knot, for every $k=1, \ldots, p-1$. Furthermore, every diagram $E_{p, p}^{k}$ has exactly $2 p-3$ negative crossings, and so, by Lemma 2.2 we have

$$
H^{i}\left(E_{p, p}^{k}\right) \text { is trivial for every } i<2 p-3 .
$$

Together with the long exact sequences (8), this gives

$$
H^{i}\left(D_{p, p}\right)=H^{i}\left(D_{p, p-1}\right) .
$$

Finally, by using (2) we have

$$
\begin{aligned}
H^{i, j}\left(D_{p, p-1}\right) & =\mathcal{H}^{i, j+(p-1)^{2}}\left(T_{p, p-1}\right) \\
& =\mathcal{H}^{i, j+p(p-2)+1}\left(T_{p-1, p}\right)=H^{i, j+1}\left(D_{p-1, p}\right),
\end{aligned}
$$

which gives (6).

\subsection{Proof of Proposition 3.5}

First of all, since $K=T_{p, q}$ is a positive braid knot whose regular diagram $D_{p, q}$ is the closure of the braid $\left(\sigma_{1} \sigma_{2} \ldots \sigma_{p-1}\right)^{q}$ with $(p-1) q$ (positive) crossings, we have that $\mathcal{H}^{4,(p-1)(q-1)+5}(K)=H^{4,6-p}\left(D_{p, q}\right)$. So, we will "concentrate" on calculating the latter homology group, ie showing that its rank is nonzero.

If $p=q=3$, then eg by using programs for computing Khovanov homology [1;16], we obtain that $\operatorname{rank} H^{4,3}\left(D_{3,3}\right)=\operatorname{rank} \mathcal{H}^{4,9}\left(T_{3,3}\right)=1$, as wanted. If $3=p<q$, then by (5) from Theorem 3.1, we have that $H^{4,3}\left(D_{3, q}\right)=H^{4,3}\left(D_{3,4}\right)$. However, by using programs for computing Khovanov homology, we obtain that the rank of the latter group (which is equal to $\mathcal{H}^{4,11}\left(T_{3,4}\right)$ ) is equal to 1 .

Remark 4.3 In Bar-Natan's tables of knots in [2], the link $T_{3,3}$ is denoted by $6_{3}^{3}$, and the torus knot $T_{3,4}$ is isotopic to the knot $8_{19}$. For the general notation of knots and links see Rolfsen [15] and The Knot Atlas [1]. 
Now, let us move to the general case $4 \leq p \leq q$. Then if $p<q$ we can apply (4) and (6) for $i=4$, and obtain

$$
H^{4,6-p}\left(D_{p, q}\right)=H^{4,6-p}\left(D_{p, p}\right)=H^{4,7-p}\left(D_{p-1, p}\right) .
$$

If $p>4$, then we have from (4) that $H^{4,7-p}\left(D_{p-1, p}\right)=H^{4,7-p}\left(D_{p-1, p-1}\right)$. By repeating this process, we can decrease the number of strands $p$, and obtain that

$$
H^{4,6-p}\left(D_{p, p}\right)=H^{4,2}\left(D_{4,4}\right) .
$$

Finally, from (6) and (2) we have

$$
H^{4,2}\left(D_{4,4}\right)=H^{4,2}\left(D_{4,3}\right)=\mathcal{H}^{4,11}\left(T_{3,4}\right),
$$

and the last homology group, as we saw previously, is of rank 1. This concludes our proof.

\subsection{Proof of Theorem 3.3}

Since $T_{p, q}$ is a positive knot, its zeroth homology group is two dimensional and the $q-$ gradings (and consequently the $\delta$-gradings) of its two generators are $(p-1)(q-1)-1$ and $(p-1)(q-1)+1$, respectively [12]. However, from Proposition 3.5 we have that there exists a generator with $t$-grading equal to 4 and $q$-grading equal to $(p-1)(q-$ $1)+5$, and so its $\delta$-grading is equal to $(p-1)(q-1)+5-2 \cdot 4=(p-1)(q-1)-3$. Thus, we have obtained three generators of the homology of the torus knot $T_{p, q}$ which have three different values of the $\delta$-grading and hence its Khovanov homology occupies at least three diagonals.

\subsection{Proof of Theorem 3.4}

Suppose that $p=3$. Then by applying (5), we obtain that $H^{i, j}\left(D_{3, q}\right)=H^{i, j}\left(D_{3,4}\right)$ for $i=0, \ldots, 4$. If $p>3$, then by applying (4) repeatedly, we obtain $H^{i, j}\left(D_{p, q}\right)=$ $H^{i, j}\left(D_{p, p}\right)$ for $i=0, \ldots, 4$. Furthermore, by applying (6) (and then (4)) repeatedly we obtain $H^{i, j}\left(D_{p, p}\right)=H^{i, j+p-3}\left(D_{3,4}\right)$ for $i=0,1,2,3,4$. Finally, the homology of the last torus knot is well-known (see eg knot $8_{19}$ in Shumakovitch [17]) and thus we obtain the required result.

\subsection{Proof of Proposition 3.6}

As we know, for example, from the proof of Theorem 3.3, there exists a generator of the homology group $\mathcal{H}^{0,(p-1)(q-1)+1}$ and its $\delta$-grading is equal to $(p-1)(q-1)+1$. Since we have assumed that

$$
\operatorname{rank} H^{2 p-2, p}\left(D_{p, q}\right)=\operatorname{rank} \mathcal{H}^{2 p-2, p+(p-1) q}\left(T_{p, q}\right)>0,
$$


we have that there exists a generator of this homology group whose $\delta$-grading is equal to $p+(p-1) q-2(2 p-2)=(p-1)(q-1)+3-2 p$. So, we have two generators whose $\delta$-gradings differ by $2 p-2$, and hence they lie on two different diagonals between which there are $p-2$ diagonals. Hence the homological width of the torus knot $T_{p, q}$ is at least $p$.

\subsection{Proof of Lemma 3.7}

We start with the diagram $D_{p, p+1}$ and we shall use the same process as in the proof of Theorem 3.1. Namely, we obtain the long exact sequences (see (8)) for every $i=1, \ldots, p-1$ :

$$
\begin{aligned}
\cdots & \rightarrow H^{2 p-3, p-1}\left(E_{p, p+1}^{i}\right) \rightarrow H^{2 p-2, p}\left(D_{p, p+1}^{i-1}\right) \rightarrow \\
& \rightarrow H^{2 p-2, p}\left(D_{p, p+1}^{i}\right) \rightarrow H^{2 p-2, p-1}\left(E_{p, p+1}^{i}\right) \rightarrow \cdots
\end{aligned}
$$

For every $i=1, \ldots, p-1$ we can calculate explicitly the number of positive and negative crossings of $E_{p, p+1}^{i}$, and we can find explicitly the positive diagram to which $E_{p, p+1}^{i}$ is isotopic.

One can easily see that the number of negative crossings of each $E_{p, p+1}^{i}$ is $2 p-2$. Hence the number of positive crossings is $(p-1)(p+1)-i-(2 p-2)=p^{2}-2 p+1-i$. On the other hand, every $E_{p, p+1}^{i}$ for $i=1, \ldots, p-2$ is isotopic (by a sequence of $\mathrm{R} 2$ and R1 moves as explained in the proof of Lemma 4.1) to the diagram $D_{p-2, p-1}^{i-1}$, while $E_{p, p+1}^{p-1}$ is isotopic to the diagram

$$
D_{p-2, p-1}^{p-3} \bigsqcup U=D_{p-2, p-2} \bigsqcup U,
$$

where by $U$ we denote the unknot. Hence $\mathcal{H}^{l}\left(E_{p, p+1}^{i}\right)=0$ for $l<0$. Also, since $D_{p, q}^{i}$ is a positive braid knot with $p$ strands and $(p-1) q-i$ crossings, we have that $\mathcal{H}^{0,(p-3)(p-2)-(i-1) \pm 1}\left(D_{p-2, p-1}^{i-1}\right)=\mathbb{Z}$ and all other $\mathcal{H}^{0, j}\left(D_{p-2, p-1}^{i-1}\right)$ are trivial. Hence, we have that the only nontrivial part of the 0 -th homology group of $E_{p, p+1}^{i}$ is given by $\mathcal{H}^{0,(p-3)(p-2)-(i-1) \pm 1}\left(E_{p, p+1}^{i}\right)=\mathbb{Z}$ for $i=1, \ldots, p-2$, and $\mathcal{H}^{0,(p-3)(p-2)-(p-3) \pm 1 \pm 1}\left(E_{p, p+1}^{p-1}\right)=\mathbb{Z}$. Thus, for every $i=1, \ldots, p-1$, $\mathcal{H}^{0, p^{2}-5 p+4-i}\left(E_{p, p+1}^{i}\right)$ is trivial.

Finally, since the number of negative crossings of $E_{p, p+1}^{i}$ is equal to $2 p-2$, we have that $H^{2 p-3}\left(E_{p, p+1}^{i}\right)$ is trivial. Furthermore, since the number of positive crossings of $E_{p, p+1}^{i}$ is equal to $p^{2}-2 p+1-i$ we have that

$$
H^{2 p-2, p-1}\left(E_{p, p+1}^{i}\right)=\mathcal{H}^{0, p^{2}-5 p+4-i}\left(E_{p, p+1}^{i}\right),
$$

which is trivial for every $i=1, \ldots, p-1$. 
Hence from the long exact sequences (12) we obtain the required

$$
H^{2 p-2, p}\left(D_{p, p}\right)=H^{2 p-2, p}\left(D_{p, p+1}\right) .
$$

\section{Stable $s l(n)$ homology of torus knots}

\subsection{Khovanov-Rozansky ( $\operatorname{sl}(n))$ homology}

In [8], M Khovanov and L Rozansky generalized the construction of the $s l(2)$ homology from Section 2.1, to the case of $s l(n)$ specializations of HOMFLYPT polynomial, for every $n \in \mathbb{N}$. This is done by categorifying the Murakami-Ohtsuki-Yamada (MOY) calculus [11] - the generalization of the Kauffman's state model for the Jones polynomial. The main form of the construction is the same: namely, they again assigned to every diagram $D$ the cubic complex of resolutions, to each vertex (total resolution) is assigned appropriate graded vector space and to every edge of the cube, certain graded preserving map. The first difference is that we start from the oriented diagram $D$, and 0 - and 1-resolutions at a certain crossing $c$ are defined according to the sign of the crossing as on the following picture:

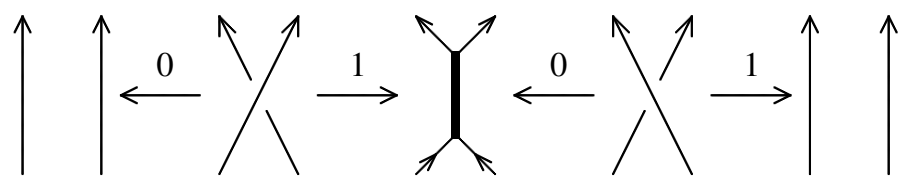

Hence, the total resolutions are in this case trivalent graphs with thick edges (edges labelled 2 in [11]), and the values assigned to them in [11] satisfy certain set of (MOY) axioms. In [8], the corresponding graded vector spaces are defined (in a rather complicated way) such that they "categorify" those axioms. Because of the complexity of this construction, the values of the $s l(n)$ homology are known only for very small set of knots.

On the other hand, the main concepts and properties are the same as in the $s l(2)$ case - the cubic complex, the mapping cone and consequently, the long exact sequence in $\operatorname{sl}(n)$ homology. If $c$ is a positive crossing of an oriented diagram $D$, then there exists long exact sequence in (unnormalized) $s l(n)$ homology:

$$
\begin{aligned}
\cdots \rightarrow H_{n}^{i-1, j+1}\left(D_{1}\right) \rightarrow H_{n}^{i, j}(D) \rightarrow & \\
& H_{n}^{i, j}\left(D_{0}\right) \rightarrow H_{n}^{i, j+1}\left(D_{1}\right) \rightarrow H_{n}^{i+1, j}(D) \rightarrow \cdots
\end{aligned}
$$

Algebraic $8 \mathcal{G}$ Geometric Topology, Volume 7 (2007) 
where $D_{i}, i=0,1$ is a diagram obtained from $D$ after resolving the crossing $c$ into an $i$-resolution. Obviously, in these long exact sequence we will always have diagrams of knots which also have trivalent vertices and thick edges, and the Khovanov-Rozansky prescription also assigns to them corresponding chain complexes and homology groups We call such diagrams, the generalized regular diagrams. As in the $s l(2)$ case, the generalized regular diagrams with only positive crossings have trivial homology groups in negative homological degrees (since the chain groups in these degrees are trivial).

For more details about the $\operatorname{sl}(n)$ homology, we refer the reader to Khovanov and Rozansky [8].

\subsection{Stability of $s l(n)$ homology of torus knots}

As we saw in Section 3.3, we have proved the existence of stable Khovanov homology for torus knots. Also, in [3] the existence of stable $s l(n)$ homology for torus knots is conjectured. However, in the course of proving the stability property in the $s l(2)$ case (Theorem 3.1 (4)) the basic ingredient is the long exact sequence in homology together with the form of the cube of resolutions. Since the analogous long exact sequence exists for $s l(n)$ homology (it is again the mapping cone), we can repeat the major part of the process. The long exact sequence in the case of $s l(n)$ homology is

$$
\begin{aligned}
\cdots \rightarrow H_{n}^{i-1, j+1}\left(D_{1}\right) \rightarrow H_{n}^{i, j}(D) \rightarrow & \\
& H_{n}^{i, j}\left(D_{0}\right) \rightarrow H_{n}^{i, j+1}\left(D_{1}\right) \rightarrow H_{n}^{i+1, j}(D) \rightarrow \cdots
\end{aligned}
$$

where $D_{i}, i=0,1$ is obtained from $D$ after resolving the positive crossing $c$ into an $i$-resolution. Note that one of the diagrams $D_{i}$ is not a planar projection of a knot since it contains one thick edge. In the case that we are interested in (torus knots and positive knots), the diagram $D_{1}$ is the one which has one thick edge (for the details and notation see Section 5.1 and Khovanov and Rozansky [8]).

As in the $\operatorname{sl}(2)$ case, we shall prove the following:

Theorem 5.1 Let $p$ and $q$ be integers such that $2 \leq p<q$. Then for every $j \in \mathbb{Z}$,

$$
H_{n}^{i, j}\left(D_{p, q-1}\right)=H_{n}^{i, j}\left(D_{p, q}\right), \quad \text { for } i<p+q-3
$$


From this formula, we conclude the existence of the limit:

$$
\begin{aligned}
P_{k}^{n}(t, q) & =\lim _{l \rightarrow \infty} \sum_{i, j \in \mathbb{Z}} t^{i} q^{j} \operatorname{dim} H_{n}^{i, j}\left(D_{k, l}\right) \\
& =\lim _{l \rightarrow \infty} \sum_{i, j \in \mathbb{Z}} t^{i} q^{j} q^{(n-1)(k-1) l} \operatorname{dim} \mathcal{H}_{n}^{i, j}\left(T_{k, l}\right) \\
& =\lim _{l \rightarrow \infty} q^{(n-1)(k-1) l} P^{n}\left(T_{k, l}\right)(t, q),
\end{aligned}
$$

for every $k$, where $P^{n}\left(T_{k, l}\right)(t, q)$ is the Poincaré polynomial of the chain complex assigned to $T_{k, l}$ by $\operatorname{sl}(n)$ homology. In other words, we obtain:

Theorem 5.2 There exists stable $\operatorname{sl}(n)$ homology for torus knots.

So, we are left with proving Theorem 5.1.

Proof of Theorem 5.1 Again, as in the proof of Theorem 3.1 in Section 4, we start from the diagram $D_{p, q}$ of the torus knot $T_{p, q}$, and we resolve the crossing $c_{p-1}=(p-1,1)$. We denote the diagram obtained by the 0 -resolution by $D_{p, q}^{1}$, and the diagram obtained by the 1 -resolution by $\bar{E}_{p, q}^{1}$. Then we have the following long exact sequence:

(16) $\cdots \rightarrow H_{n}^{i-1, j+1}\left(\bar{E}_{p, q}^{1}\right) \rightarrow H_{n}^{i, j}\left(D_{p, q}\right) \rightarrow H_{n}^{i, j}\left(D_{p, q}^{1}\right) \rightarrow H_{n}^{i, j+1}\left(\bar{E}_{p, q}^{1}\right) \rightarrow \cdots$

We continue the process, by resolving the crossings $c_{l}=(l, 1), l=p-2, \ldots, 1$ of the diagram $D_{p, q}^{p-1-l}$ and we denote the 0 - and 1-resolution obtained, by $D_{p, q}^{p-l}$ and $\bar{E}_{p, q}^{p-l}$, respectively. Then we have the following long exact sequence for $l=2, \ldots, p-1$ :

$$
\cdots \rightarrow H_{n}^{i-1, j+1}\left(\bar{E}_{p, q}^{l}\right) \rightarrow H_{n}^{i, j}\left(D_{p, q}^{l-1}\right) \rightarrow H_{n}^{i, j}\left(D_{p, q}^{l}\right) \rightarrow H_{n}^{i, j+1}\left(\bar{E}_{p, q}^{l}\right) \rightarrow \cdots
$$

Like in the $s l(2)$ case, we shall prove the following:

Lemma 5.3 The homology group $H_{n}^{i}\left(\bar{E}_{p, q}^{l}\right)$ is trivial for every $l<p$ and $i<p+q-3$.

This lemma, together with the above long exact sequences (16) and the fact that $D_{p, q}^{p-1}=D_{p, q-1}$ gives Theorem 5.1.

Thus, to finish the proof, we are left with proving Lemma 5.3. We will use more or less the same approach as in Lemma 4.1. Let $C_{n}\left(\bar{E}_{p, q}^{l}\right)$ be the chain complex assigned by $s l(n)$ link homology [8] to $\bar{E}_{p, q}^{l}$. Then $H_{n}^{i}\left(\bar{E}_{p, q}^{l}\right)=H^{i}\left(C_{n}\left(\bar{E}_{p, q}^{l}\right)\right)$. Note that in the $\operatorname{sl}(n)$ case, a complex $C_{n}$ is assigned to generalized regular diagrams, ie to regular diagrams where we also allow trivalent vertices and thick edges.

Algebraic $8 \mathcal{G}$ Geometric Topology, Volume 7 (2007) 
Since $\bar{E}_{p, q}^{l}$ has only positive crossings, its homology groups are trivial for negative homological degrees. However, we will show that

$$
C_{n}\left(\bar{E}_{p, q}^{l}\right) \sim D_{n}[p+q-3],
$$

where $D_{n}$ is a complex such that all its chain groups $D_{n}^{i}$ are trivial for $i<0$ (in fact, we will define $D_{n}$ as the direct sum of the complexes of the form $C_{n}\left(D_{\Gamma}^{i}\right)$, where $D_{\Gamma}^{i}$ 's are the generalized regular diagrams whose all crossings are positive). Here, by $\sim$ we denote a quasi-isomorphism, which implies that the two complexes have isomorphic homology groups. Then (17) implies that $H_{n}^{i}\left(\bar{E}_{p, q}^{l}\right)=H^{i}\left(C_{n}\left(\bar{E}_{p, q}^{l}\right)\right)$ is trivial for $i<p+q-3$. Like in Lemma 4.1, we have that the two lower strands (thin edges) of the $\bar{E}_{i}$ part, will form at least two crossings with each other (corresponding to an R1 move in the proof of Lemma 4.1) and both of them will have at least $p+q-5$ over- or undercrossings with the same strand (corresponding to an R2 move in the proof of Lemma 4.1). We will show that in each of these cases we can "shift" up our complex by one homological degree, and thus obtain (17). In order to prove this we will use the following fact ("cancellation principle" for chain complexes): if we quotient the chain complex $\mathcal{C}$ by an (arbitrary) acyclic subcomplex $\mathcal{C}^{\prime}$ (ie a subcomplex with trivial homology), then the quotient complex $\mathcal{C} / \mathcal{C}^{\prime}$ is quasi-isomorphic to the complex $\mathcal{C}$, and so they have isomorphic homology groups (see eg [2, Lemma 3.7]).

Untwisting an R1 move First, let us work with the analog of the R1 move. Let $\bar{D}$ be the diagram that contains the following diagram as a subdiagram:

$$
\text { D: }
$$

Then the chain complex $C_{n}(\bar{D})$ associated to $\bar{D}$ is the mapping cone of a certain homomorphism $f: C_{n}\left(\bar{D}_{0}\right) \rightarrow C_{n}\left(\bar{D}_{1}\right)\{-1\}$, where $\bar{D}_{0}$ and $\bar{D}_{1}$ are the 0 - and 1resolutions, respectively, of the crossing of $D$ [8]. In other words, they look the same as the diagram $\bar{D}$ except that its subdiagram $D$ is replaced by $D_{0}$ and $D_{1}$, respectively:

$$
D_{0}: D_{1}: \underbrace{\top}
$$

In other words, the complex associated to $\bar{D}$ is the total complex of the complex given by $C_{n}\left(\bar{D}_{0}\right) \rightarrow C_{n}\left(\bar{D}_{1}\right)[1]\{-1\}$. Furthermore, by the "categorification" of one of the MOY axioms [8, Proposition 30] we have that

$$
C_{n}\left(\bar{D}_{1}\right) \cong C_{n}\left(\bar{D}_{0}\right)\{1\} \oplus C_{n}\left(\bar{D}_{0}\right)\{-1\} .
$$

Algebraic $8 \mathcal{G}$ Geometric Topology, Volume 7 (2007) 
We also have that the projection of $f$ to the first summand is an isomorphism by invariance under the Reidemeister IIa move [8], and hence the complex $C_{n}(\bar{D})$ is quasi-isomorphic to $C_{n}\left(\bar{D}_{0}\right)[1]\{-2\}$ (by the cancellation principle). So, the last two complexes have isomorphic homology groups. Thus, we can "untwist" the crossing involving two strands that are connected to the same thick edge (the analog of an R1 move in the $s l(2)$ case) by shifting the complex of the diagram obtained up by one in homological degree, as required.

Untwisting an R2 move Hence, after untwisting the two crossings of $\bar{E}_{p, q}^{l}$ that were resolved in the $\operatorname{sl}(2)$ case by the R1 move, we are left with a diagram of the following form:

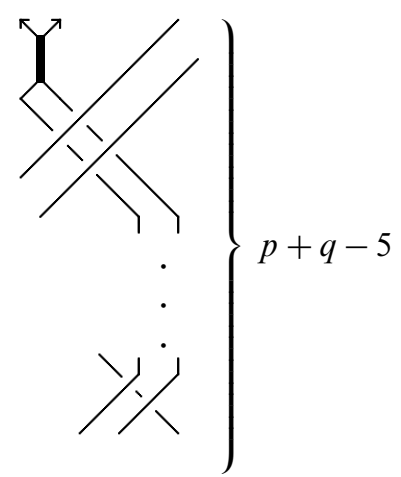

In other words, we have two neighboring strands that are both connected to the same thick edge, and both go over or both go under $p+q-5$ strands. We will show that the complex corresponding to this diagram is quasi-isomorphic to the complex of diagrams whose crossings are all positive, shifted up in homological degree by $p+q-5$. Let $\bar{D}$ be a positive diagram that contains the following diagram as a subdiagram:

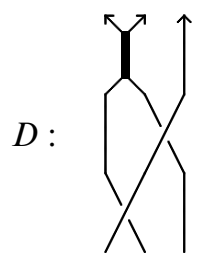

Denote by $D_{0}, D_{1}^{1}, D_{1}^{2}$ and $D_{2}$ the resolutions obtained from $D$ by resolving its two crossings, according to the following pictures:

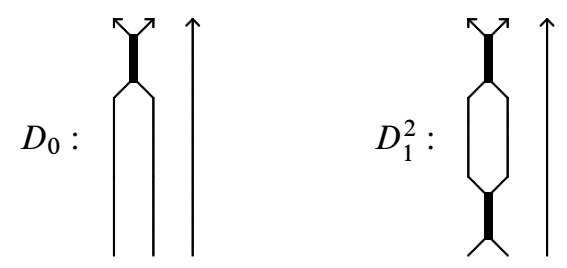

Algebraic 83 Geometric Topology, Volume 7 (2007) 

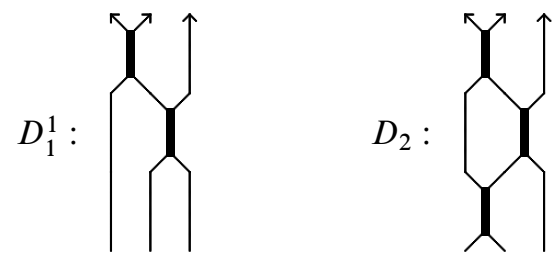

Denote by $\bar{D}_{0}, \bar{D}_{1}^{1}, \bar{D}_{1}^{2}$ and $\bar{D}_{2}$ the diagrams obtained from $\bar{D}$, after replacing the subdiagram $D$ by $D_{0}, D_{1}^{1}, D_{1}^{2}$ and $D_{2}$, respectively. Then we have that the complex $C_{n}(\bar{D})$ associated to the diagram $\bar{D}$ is the total complex of the following complex of complexes:

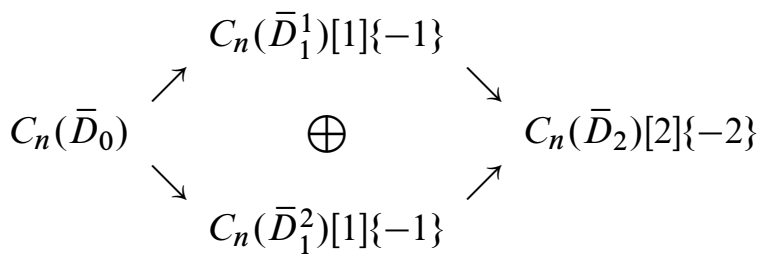

Like previously, we have that

$$
C_{n}\left(\bar{D}_{1}^{2}\right) \cong C_{n}\left(\bar{D}_{0}\right)\{1\} \oplus C_{n}\left(\bar{D}_{0}\right)\{-1\},
$$

and the projection of the map from $C_{n}\left(\bar{D}_{0}\right)$ onto the first summand of $C_{n}\left(\bar{D}_{1}^{2}\right)[1]\{-1\}$ is an isomorphism. Hence, again we can quotient by an acyclic complex and obtain that $C_{n}(\bar{D})$ is quasi-isomorphic to the total complex of the following:

$$
\begin{array}{ccc}
C_{n}\left(\bar{D}_{1}^{1}\right)[1]\{-1\} & \\
\bigoplus & \searrow \\
C_{n}\left(\bar{D}_{0}\right)[1]\{-2\} & \nearrow & C_{n}\left(\bar{D}_{2}\right)[2]\{-2\} \\
& &
\end{array}
$$

Also, we have that

$$
C_{n}\left(\bar{D}_{2}\right) \cong C_{n}\left(\bar{D}_{0}\right) \oplus C_{n}\left(\bar{D}_{3}\right),
$$

by the "categorification" of the last MOY [8, Proposition 33] and that the map from the second summand of (18) to the first summand of (20) is an isomorphism by invariance under the Reidemeister III move [8]. Here by $\bar{D}_{3}$ we denoted the diagram that is the same as $\bar{D}$ with the subdiagram $D$ replaced by the following diagram $[8 ; 11]$ :

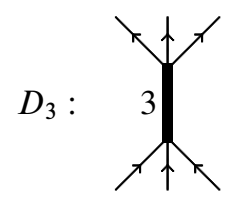


Hence $C_{n}(\bar{D})$ is quasi-isomorphic to the total complex of

$$
C_{n}\left(\bar{D}_{1}^{1}\right)[1]\{-1\} \rightarrow C_{n}\left(\bar{D}_{3}\right)[2]\{-2\} .
$$

On the other hand the complex $C_{n}\left(\bar{D}_{3}\right)$ is quasi-isomorphic to the total complex of both of the two complexes

and

$$
\begin{aligned}
& C_{n}\left(\bar{D}_{0}\right)[-1] \rightarrow C_{n}\left(\bar{D}_{2}\right), \\
& C_{n}\left(\bar{D}_{0}^{\prime}\right)[-1] \rightarrow C_{n}\left(\bar{D}_{2}^{\prime}\right),
\end{aligned}
$$

where $\bar{D}_{0}^{\prime}$ and $\bar{D}_{2}^{\prime}$ are the diagrams obtained from $\bar{D}$ after replacing $D$ by following two diagrams, respectively:

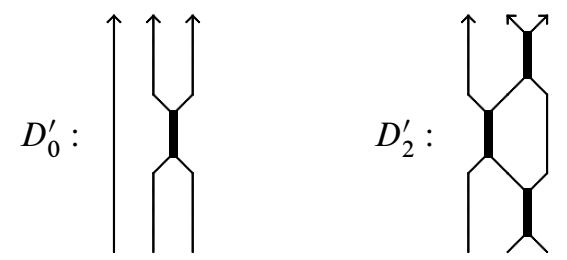

Thus, the total complex of (19) (and hence $C_{n}(\bar{D})$ ) is quasi-isomorphic to the total complex of the following complex:

$$
\begin{array}{ccc}
C_{n}\left(\bar{D}_{1}^{1}\right)[1]\{-1\} & \\
\bigoplus & \searrow & C_{n}\left(\bar{D}_{2}^{\prime}\right)[2]\{-2\} \\
C_{n}\left(\bar{D}_{0}^{\prime}\right)[1]\{-2\} & \nearrow &
\end{array}
$$

Thus, if we denote by $D_{n}$ the above complex shifted down in homological degree by 1 , then we have that $C_{n}(\bar{D}) \sim D_{n}[1]$, and all homology groups of $D_{n}$ are in nonnegative homological degrees, as required (the crossings in three diagrams appearing in (22) are all positive). We can now iterate the argument for each instance when an R2 move would occur in the $s l(2)$ case, since the two lower rightmost strands are both connected to the same thick edge in all three diagrams $D_{1}^{1}, D_{0}^{\prime}$ and $D_{2}^{\prime}$, and hence we can continue the process like with the initial diagram $D$. Completely analogously, we obtain the same result for the diagram $D$ of the following form:

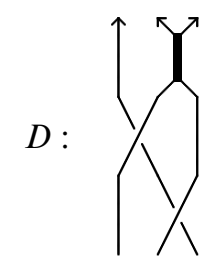

Algebraic 83 Geometric Topology, Volume 7 (2007) 
Thus, we obtain the required shift in homological degree.

\section{References}

[1] D Bar-Natan, The Knot Atlas Available at http://www.math.toronto.edu/ drorbn/Katlas/

[2] D Bar-Natan, On Khovanov's categorification of the Jones polynomial, Algebr. Geom. Topol. 2 (2002) 337-370 MR1917056

[3] N M Dunfield, S Gukov, J Rasmussen, The superpolynomial for knot homologies, Experiment. Math. 15 (2006) 129-159 MR2253002

[4] V F R Jones, A polynomial invariant for knots via von Neumann algebras, Bull. Amer. Math. Soc. (N.S.) 12 (1985) 103-111 MR766964

[5] L H Kauffman, Knots and physics, third edition, Series on Knots and Everything 1, World Scientific Publishing Co., River Edge, NJ (2001) MR1858113

[6] M Khovanov, A categorification of the Jones polynomial, Duke Math. J. 101 (2000) 359-426 MR1740682

[7] M Khovanov, Patterns in knot cohomology. I, Experiment. Math. 12 (2003) 365-374 MR2034399

[8] M Khovanov, L Rozansky, Matrix Factorizations and link homology arXiv: math.QA/0401268

[9] E Lee, On Khovanov invariant for alternating links arXiv:math.GT/0210213

[10] E Lee, The support of the Khovanov's invariants for alternating knots arXiv: math.GT/0201105

[11] H Murakami, T Ohtsuki, S Yamada, Homfly polynomial via an invariant of colored plane graphs, Enseign. Math. (2) 44 (1998) 325-360 MR1659228

[12] J Rasmussen, Khovanov homology and slice genus arXiv:math.GT/0402131

[13] J Rasmussen, Khovanov-Rozansky homology of two-bridge knots and links arXiv: math.GT/0508510

[14] J Rasmussen, Knot polynomials and knot homologies, from: "Geometry and topology of manifolds", Fields Inst. Commun. 47, Amer. Math. Soc., Providence, RI (2005) 261-280 MR2189938

[15] D Rolfsen, Knots and links, Mathematics Lecture Series 7, Publish or Perish, Berkeley, CA (1976) MR0515288

[16] A Shumakovitch, KhoHo: a program for computing Khovanov homology Available at http://www.geometrie.ch/KhoHo/

[17] A Shumakovitch, Torsion of the Khovanov homology arXiv:math.GT/0405474 
[18] M Stošić, Properties of Khovanov homology for positive braid knots arXiv: math.QA/0511529

[19] P Turner, Calculating Bar-Natan's characteristic two Khovanov homology arXiv: math.GT/0411225

[20] O Viro, Remarks on the Definition of the Khovanov homology arXiv: math.GT/0202199

[21] B Webster, Kr.m2 Available at http://katlas.math.toronto.edu/wiki/ User: Ben/KRhomology

Instituto de Sistemas e Robótica and CAMGSD, Instituto Superior Técnico, TU Lisbon Av Rovisco Pais 1, 1049-001 Lisbon, Portugal

mstosic@math.ist.utl.pt

Received: 24 September 2006 